\title{
Biological Carcinogenesis, Non-Viral
}

National Cancer Institute

\section{Source}

National Cancer Institute. Biological Carcinogenesis, Non-Viral. NCI Thesaurus. Code C26018.

Studies of the role of non-viral biological agents as factors or cofactors in the etiology of human and animal cancer. 